\title{
L'efficacia del Customer Relationship Management nei mercati dei servizi tecnologici: il caso di una media impresa italiana*
}

\author{
di Gian Luca Gregori, Andrea Perna e Andrea Sabatini
}

L'analisi dell'efficacia dei sistemi CRM è un tema ancora fortemente dibattuto ed oggetto di numerosi studi nelle discipline di management (Elmuti et al., 2009; Perna-Baraldi, 2014). In tal senso, risulta interessante verificare le ragioni per cui numerosi progetti CRM intrapresi da imprese - soprattutto quelle operanti in mercati business-to-business e focalizzate sulla fornitura di servizi tecnologici più o meno complessi - non generino benefici attesi e, quindi, falliscano successivamente $o$ addirittura durante la fase implementativa.

Il principale obiettivo del presente lavoro è quello di comprendere i fattori e le dimensioni che impattano sul livello di efficacia del CRM. Gli autori sono interessati, in modo particolare, ad investigare il complesso rapporto tra efficacia del CRM ed aspettative che le imprese manifestano circa il raggiungimento di benefici tangibili durante le fasi di gestione del cliente. Metodologicamente gli autori fanno riferimento all'uso del caso di studio qualitativo come strategia di ricerca.

Nello specifico viene proposto il caso del gruppo Loccioni, impresa operante nel settore della produzione e fornitura di macchine di collaudo e servizi integrati ad alto contenuto tecnologico. L'azienda si caratterizza per aver avviato un processo di implementazione di un software CRM che ha comportato sensibili effetti a livello organizzativo e strategico. Inoltre, interessante notare come l'uso del sistema abbia a sua volta generato effetti inaspettati, non sempre prevedibili e variabili rispetto le diverse tipologie di relazioni aziendali.

Lo studio propone diversi tipi di contributi. Dal lato empirico, il caso evidenzia ed illustra le problematiche relative al miglioramento di processi gestionali legati alla implementazione ed uso del CRM in un settore abbastanza particolare come quello della fornitura di servizi altamente tecnologici. Dal punto di vista teorico, si

\footnotetext{
* Il lavoro è frutto del comune impegno dei tre Autori. Tuttavia, i $\S \S 1$ e 6 sono da attribuire a Gian Luca Gregori, i §§ 3, 4 e 5 ad Andrea Perna, il § 2 ad Andrea Sabatini.
}

Economia e diritto del terziario (ISSNe 1972-5256), 2017, 2 
vuole contribuire nel marketing b2b e dei servizi riguardo al tema della efficacia di azioni di marketing in contesti di mercato altamente complessi.

Parole chiave: CRM, business to business, case study, efficacia

The effectiveness of CRM systems is still highly debated in management and marketing studies. The goal of this paper is to shed light on what the main factors and dimensions that affect the effectiveness of CRM systems are. Moreover the Authors are interested to investigate on what is the relationship between CRM adoption and expected performances. Methodologically, we refer to the use of a single case study for showing how CRM has been implemented, adopted and critically re-organized by a medium-sized b2b company. Our findings clearly show that CRM systems are strongly influenced by social as well as organizational dimensions which might or not might link to the complexity of the technology implemented. Moreover, the case shows that positive performances of CRM vary considerably depending on the attitudes and behaviours of the investigated users considered. The paper identifies future research avenues in the field of CRM in business-to-business markets.

Keywords: CRM, business to business, effectiveness, industrial relations

JEL Classification: M31

\section{Introduzione}

Nella letteratura scientifica su tematiche di management, il CRM (Customer Relationship Management) - identificabile in senso lato come uno strumento di Information Technology (IT) a supporto dello sviluppo di alcuni processi di marketing - viene considerato potenzialmente utile per la gestione della customer knowledge e, più in generale, per la gestione delle relazioni con i clienti (Bucic-Ngo-Sinha, 2017; Kalaignanam-Varadarajan, 2012; Payne and Frow, 2005). Numerosi studi evidenziano i vantaggi derivanti dall'impiego di tali sistemi; per esempio, la possibilità di estendere e condividere all'interno dell'organizzazione il patrimonio conoscitivo del mercato e permettere la fruibilità di una migliore conoscenza dei clienti. Tali vantaggi risultano evidenti in particolar modo per i mercati business-to-business ad alta tecnologia, proprio perché consentono una gestione integrata del processo di co-creazione della tecnologia e di integrazione tra entità. Senza dubbio le imprese operanti nei mercati industriali potrebbero ottenere benefici oggettivi derivanti dall'uso di tecnologie CRM per via della presenza di meccanismi interattivi (Håkansson-Snehota, 1995) che richiedono la gestione di dati ed informazioni complessi. In altre parole, la particolare 
struttura network-based del mercato industriale (operatori concentrati, presenza di importanti volumi di scambio, continuità degli scambi, difficoltà di sostituzione della controparte, ecc.) comporta una profonda attenzione verso la gestione dell'economia della relazione con il cliente che si basa sulla presenza di molteplici e rilevanti episodi interattivi. Un sistema CRM permetterebbe la raccolta, gestione e controllo di informazioni critiche e complesse diminuendo il rischio di perdere importanti "pezzi" di informazioni riguardanti le stesse relazioni di business.

Tra i temi analizzati, comunque, si distinguono per un grande interesse quelli relativi alla comprensione ed all'analisi dell'efficacia del CRM. Tale topic sembra costituire un ambito di ricerca attuale, ma non molto sviluppato (Elmuti et al., 2009; Perna-Baraldi, 2014) nonostante, secondo accademici ed operatori del mercato, il numero di progetti CRM destinati al fallimento sia costantemente in crescita.

Il presente lavoro si pone, come obiettivo principale proprio quello di comprendere i fattori che influenzano l'efficacia del CRM in contesti di mercato caratterizzati dallo scambio di soluzioni e servizi altamente complessi. Gli autori sono interessati, in modo particolare, ad investigare il complesso rapporto tra efficacia del CRM ed aspettative manifestate dai suoi utilizzatori: in questo studio può essere utile osservare in proposito che l'efficacia viene intesa come la capacità del CRM di produrre benefici tangibili per l'intera organizzazione.

Il paper è volto a contribuire al corrente dibattito sull'efficacia dei sistemi CRM mediante la discussione delle domande di ricerca di seguito presentate:

1. Quali sono i fattori che influenzano l'efficacia del CRM in mercati caratterizzati dallo scambio di servizi tecnologici?

2. Come varia la percezione degli utilizzatori del CRM rispetto alla sua efficacia?

Metodologicamente gli autori propongono uno studio qualitativo basato sul caso del gruppo Loccioni - impresa operante nella fornitura di soluzioni e servizi di misura e collaudo per componentistica ad elevato contenuto tecnologico - che, a partire dal 2006, ha intrapreso un investimento in un sistema di CRM.

Il lavoro offre nella sezione successiva un'analisi della letteratura che approfondisce il tema della complessità gestionale delle relazioni con i clienti e degli elementi che influenzano l'efficacia del CRM. La sezione 3 presenta alcune note metodologiche, nella sezione 4 viene presentato il caso di studio, mentre le sezioni 5 e 6 sono dedicate rispettivamente all'analisi del caso ed alle conclusioni ed implicazioni manageriali. 


\section{Analisi della letteratura}

\subsection{La complessità ed il dinamismo delle relazioni di business}

Gli studi condotti dalla scuola di pensiero IMP group ${ }^{1}$ (Industrial Marketing \& Purchasing) hanno chiaramente evidenziato che le relazioni industriali (Håkansson-Snehota, 1995; Ford et al., 2003-2006) si caratterizzano per una elevata dinamicità e complessità; infatti, queste rappresentano il veicolo mediante il quale le imprese scambiano risorse e sviluppano attività. Ma soprattutto le relazioni - per esempio quelle tra fornitori e clienti operanti in mercati dove l'oggetto di scambio sono servizi ad alto contenuto tecnologico generano forti interdipendenze (Håkansson and Snehota, 1995; Ford et al., 1998), che possono condurre ad effetti positivi (si pensi ai meccanismi di apprendimento reciproco che permettono di generare valore), ma anche costituire un vero e proprio problema limitante lo sviluppo futuro della relazione stessa (per esempio, i casi in cui il cliente o il fornitore eserciti forti pressioni sulla controparte o manifesti un eccessivo potere durante i momenti di interazione).

La complessità ed il dinamismo appaiono essere dimensioni molto difficili da prevedere e, quindi, $i$ tentativi di riuscire a prefigurare azioni e strategie per contenere i possibili problemi di gestione della relazione risultano spesso vani. In altri termini, la creazione, lo sviluppo e la gestione delle relazioni risultano essere un "complicato esercizio" per tutte le organizzazioni, a prescindere dalle dimensioni aziendali e dai settori industriali in cui esse operano (Håkansson and Ford, 2002; Gadde et al., 2012; La Rocca et al., 2013): in proposito è stato contestualmente rilevato che "the development of a customer relationship requires coordination of the interactions between a customer and a supplier. This coordination entails costs and problems for both companies and it limits their freedom to coordinate with others" (Ford et al., 2011: 53). Un'ulteriore riflessione deve partire dalla constatazione che le imprese non operano in isolamento, ma, al contrario, sono fortemente dipendenti dal portafoglio di relazioni create con i vari attori del business network, nel quale operano. In effetti,

\footnotetext{
${ }^{1}$ Il gruppo di ricerca internazionale IMP - costituitosi agli inizi degli anni Settanta nel Nord Europa - si focalizza sullo studio dei complessi processi inter-organizzativi tra imprese (sviluppo nuovi prodotti ed innovazione, acquisti, gestione dei clienti, ecc.) mediante l'adozione di un approccio interattivo. In particolare, lo studio delle relazioni di business intese come quasi-organizzazioni ossia strutture che determinano ed influenzano i processi tra imprese appare come il principale interesse di ricerca perseguito. Per approfondimenti circa i temi di ricerca ed i risultati dei principali studi condotti si rimanda al sito web www.impgroup.org.
} 
molti studi a partire dagli anni Ottanta (Ford, 1980; Håkansson et al., 2009) hanno verificato che la singola relazione tra due attori è necessariamente parte di un contesto "network" molto più ampio ed esteso, rispetto allo stesso. Ed il vero problema consiste nella dinamicità del network; infatti i profondi e repentini cambiamenti che caratterizzano le singole relazioni - in virtù degli scambi che avvengono tra gli attori - influenzano anche altre relazioni non direttamente collegate agli attori coinvolti.

Questa premessa è utile per introdurre alcune dimensioni principali che influenzano i rapporti di business tra imprese e quindi le relazioni che esse sviluppano.

Håkansson-Snehota (1995) ne identificano alcune interessanti che derivano da osservazioni empiriche condotte in settori industriali altamente complessi, di seguito analizzati:

- le relazioni si originano al fine di permettere alle parti coinvolte negli scambi di risolvere problematiche di tipo organizzativo, processuale, gestionale, ecc.; in sostanza, le relazioni sono indispensabili in quanto vettori dello scambio di risorse tangibili e intangibili;

- le relazioni sono "entità" che si influenzano in maniera reciproca in quanto fortemente interconnesse tra esse. Le relazioni non sembrano essere strutture statiche, ma al contrario piuttosto dinamiche;

- a seguito delle interdipendenze che si generano nei rapporti di business, emerge un altro importante elemento che influenza il divenire delle relazioni e che si riconduce alla presenza di adattamenti "strutturali"; tali adattamenti appaiono durante lo sviluppo della relazione, in quanto le parti coinvolte sono alla costante ricerca di benefici reciproci. Come evidenziato, risulta fondamentale che gli scambi di risorse tra gli attori coinvolti nella relazione siano bilanciati e generino benefici; questo implica la nascita di meccanismi adattivi rispetto agli elementi scambiati e soprattutto rispetto allo "scopo" della relazione. La nozione di adattamento, in sostanza, costituisce un elemento intrinseco di ogni relazione di business;

- difficilmente le relazioni di business si limitano ad influenzare solo le parti direttamente coinvolte nello scambio; esse, proprio perché appartengono a strutture network, esercitano effetti su una moltitudine di altre relazioni collegate direttamente o indirettamente a quelle "diadiche";

- le relazioni sono il risultato di esperienze e di differenti modus operandi delle parti coinvolte, ciò significa che le relazioni sono influenzate dalle esperienze passate e si modificano continuamente a seguito degli eventi futuri che caratterizzeranno i business degli stessi attori. 
Per questo motivo, gestire le relazioni richiede l'adozione una prospettiva di lungo periodo, anziché un approccio tattico.

Infine, appare interessante evidenziare che la complessità di una relazione di business, anche se difficile da quantificare, possa essere verificata tramite il ricorso ad alcuni parametri di natura quali-quantitativa. Il numero e la frequenza dei contatti tra gli attori (Ford-Rosson, 1982; Cunningham-Turnbull, 1982), la tipologia di prodotto e servizio oggetto di scambio, la dimensione dei volumi di scambi effettuati sono considerati fattori utili alla comprensione della dinamicità delle relazioni in atto.

Specialmente in mercati in cui gli oggetti di scambio sono soluzioni e servizi industriali (Ford et al., 2011), si avverte una sensibile crescita della complessità nel gestire il cliente proprio a causa della natura derivata della domanda che, probabilmente, richiede una veloce reazione del fornitore nel rispondere alle improvvise esigenze dei clienti finali.

\subsection{Eterogeneità delle relazioni di business e relativi problemi gestio- nali}

Nel caso specifico delle relazioni fornitore-cliente, vari contributi di ricerca, negli anni, sono stati realizzati nel tentativo di effettuare una classificazione delle stesse. Dati i caratteri di complessità e dinamismo, appare scontato considerare che le singole relazioni con $i$ clienti andrebbero gestite in rapporto al business network di riferimento e non in maniera isolata. Pertanto si evidenzia un'evoluzione metodologica che si fonda sul passaggio dall'analisi della singola relazione verso l'analisi "sistematica" del portafoglio delle relazioni con i clienti del fornitore (Fiocca, 1982; Turnbull-Zolkiewski, 1997).

Emerge quindi per le imprese la necessità di adottare approcci integrati alla gestione delle relazioni di business con il cliente; riportare un focus dedicato, quindi, alla identificazione di dimensioni e fattori chiave sui quali concentrare gli sforzi economici e strategici. Ford $(1998 ; 2006)$ individua alcuni interessanti elementi, quali ad esempio il livello di importanza strategica del cliente rispetto il fornitore, la coerenza del cliente rispetto alle risorse e alle attività gestite dal fornitore, il livello di internazionalizzazione del cliente-mercato, il rapporto tra costi di gestione del cliente e valore generato rispetto un'ottica di lungo periodo.

Ford (2006) fornisce un contributo interessante rispetto ai potenziali compiti e mansioni da svolgere - in special modo a contributo della funzione vendite e marketing - al fine di gestire la complessità gestionale. I risultati 
derivanti da intensi e prolungati periodi di osservazione diretta delle dinamiche clienti-fornitori in settori quali la fornitura di servizi a valore aggiunto, hanno consentito di delineare alcuni risultati. Innanzitutto, le risorse deputate a gestire il cliente dovrebbero «apprendere l'importanza dei processi interattivi» come elementi basilari per effettuare una "sana" gestione della relazione stessa; la capacità di limitare i potenziali conflitti e la gestione del potere assumono una straordinaria rilevanza in tal senso. Ciò diventa imprescindibile da un'attenta analisi delle caratteristiche del cliente circa la sua storia, la propensione alla pianificazione di investimenti di medio e lungo termine in ricerca e sviluppo, la posizione di mercato e finanziaria, la percezione generale riscontrata dal mercato di riferimento. Tali valutazioni sono fondamentali in quanto conducono a configurare una customer knowledge utile verso lo sviluppo di robuste attività di segmentazione e categorizzazione del portafoglio clienti.

Alla luce delle problematiche gestionali qui definite, un interessante quesito verte su quali possano essere i possibili processi da sviluppare per gestire in maniera ottimale il cliente. Oltre ai descritti approcci strategici del fornitore - da adottare riguardo al problema della complessità e dinamicità - sembra assumere una certa rilevanza l'uso di sistemi di Information Technology (IT), quali quelli derivanti dall'applicazione dei principi del relationship marketing (Grönroos, 1994) e riconosciuti sotto l'acronimo CRM (Customer Relationship Management).

La letteratura sul tema in proposito appare vasta, soprattutto a partire dagli inizi degli anni Novanta, quando furono pubblicati i primi lavori che in maniera esplicita analizzavano le potenzialità derivanti dall'uso di sistemi informatici per gestire i dati sui clienti (Stone-Woodcock-Wilson, 1996), mediante implementazione dei primi data base aziendali. Una emergente scuola di pensiero dedicata a tali studi e ricerche si è sviluppata in Scandinavia, conosciuta come Nordic School of Service Marketing (Gummesson-Lehtinen-Grönroos, 1997). L'essenza ed il principale contributo di tale scuola si riferisce all'importanza ed alla rilevanza del cliente in una prospettiva di gestione multilaterale e continua nel tempo, ne risulta quindi che «The very essence of relationship marketing provides the conditions for connecting to relevant IT tools: "Relationship marketing is attracting, maintaining and in multi-service organizations enhancing customer relationships. Servicing and selling existing customers is viewed to be just as important to long-term marketing success as acquiring new customers"» (Berry, 1983: 25). 


\subsection{Fattori ed elementi che influenzano l'efficacia del CRM}

Rispetto agli obiettivi del presente lavoro è forse vano voler offrire una panoramica delle diverse accezioni che a partire dai primi anni Novanta hanno caratterizzato il fenomeno del $\mathrm{CRM}^{2}$, ma si ritiene comunque opportuno inquadrare il concetto di CRM rispetto ad alcune dimensioni principali che lo caratterizzano: gli utilizzatori del sistema, il tipo di tecnologia prescelta e l'assetto organizzativo. In un tentativo di far convergere i tre elementi in una definizione di CRM adatta al presente studio, si fa riferimento a Baraldi et al. (2013) secondo i quali «CRM systems as devices that interact with people who insert data to receive back processed information which they can use for managing customer relationships». Tale definizione appare coerente in quanto consente di verificare che il CRM richiede di combinare in maniera armoniosa e sinergica la tecnologia, gli utenti e la strategia di gestione dei clienti al fine di percepire effettivamente i benefici e di ottenere un risultato efficace.

Come accennato nella sezione introduttiva, sono molteplici gli studi condotti al fine di comprendere quali siano le circostanze che influenzano l'efficacia derivante dall'uso del CRM.

Hillebrand et al. (2011) hanno sviluppato uno studio quantitativo i cui risultati chiaramente indicano come l'efficacia del CRM dipenda dalle motivazioni che spingono il management ad adottare il sistema stesso. Per esempio, le organizzazioni che implementano il CRM in risposta a stimoli che provengono da soggetti esterni all'impresa (si pensi a stakeholders, media, consulenti, ecc.), oppure esclusivamente per imitare i concorrenti, sono spesso quelle penalizzate in quanto non riuscirebbero a ottenerne i reali benefici. Quindi, motivare l'avvio di un progetto CRM solo per conformarsi a delle pratiche attuate altrove non rappresenta un sano approccio strategico.

Rendere efficace l'uso del CRM costituisce un interessante paradigma anche in relazione alle modalità con le quali viene sviluppato il complesso processo di implementazione del sistema stesso.

Come identificato in letteratura (Bull, 2003), implementare il CRM corrisponde ad attuare una strategia olistica che prevede una costante condivisione di obiettivi tra le figure chiamate ad occuparsi di questo processo ed $\mathrm{i}$ futuri utilizzatori del sistema. Evidenzia Cambra-Fierro (2017), che il solo elemento tecnologico di per sé non costituirebbe il motivo principale degli insuccessi del CRM - anche se sono ancora considerevoli alcuni effetti che

\footnotetext{
${ }^{2}$ Per una esaustiva analisi circa la concettualizzazione del CRM si rimanda ai contributi di Payne-Frow (2005), Zablah et al. (2004).
} 
la tecnologia può esercitare sulla buona riuscita di un progetto CRM specialmente se essa non viene implementata tenendo conto della struttura organizzativa (Garrido-Moreno et al., 2014) in riferimento di tale; piuttosto, il fallimento di tale progetto dovrebbe essere inquadrato in un contesto ampio dove altre variabili soft - per esempio cultura aziendale, visione cliente-centrica dell'organizzazione, grado di propensione ad investimenti in marketing, ecc. - sono considerate in combinazione con la tecnologia. Si tratta dell'assenza di un chiaro coordinamento tra tecnologia ed aspetti organizzativi che inciderebbe negativamente sulle aspettative (Boulding et al., 2005).

Inoltre, non può sfuggire che le prassi e che le procedure sviluppate per avviare il processo implementativo del CRM costituiscono altri fondamentali elementi da considerare in rapporto all'efficacia del sistema. Rilevanti appaiono i risultati di recenti ricerche rispetto all'analisi di alcuni principali fattori che, durante l'implementazione del sistema, potrebbero esercitare una notevole influenza sul buon risultato dello stesso: il coinvolgimento dei futuri utilizzatori sin dalle prime fasi del processo di implementazione (Avlonitis-Panagopoulos, 2005); il coinvolgimento del top management aziendale anche al fine di motivare gli utenti verso l'adozione del CRM (Zablah et al., 2004); la propensione all'utilizzo di software dedicati alla gestione di informazioni critiche di mercato (Alshawi et al., 2011); l'avvio di buone pratiche di marketing durante e successivamente il periodo di implementazione del sistema (Chang et al., 2010).

Un altro elemento che emerge dall'analisi della letteratura riguardante la variabilità dei livelli di efficacia del CRM riflette la natura e la tipologia di cliente servito (Williams et al., 2016). Tali autori, pur riconoscendo il ruolo fondamentale del processo di implementazione come dimensione cardine utilizzata per giustificare spesso il fallimento dei progetti CRM, suggeriscono di adottare il CRM per gestire solo clienti "coerenti" con la strategia aziendale. In altre parole, si otterrebbe un beneficio maggiore nell'uso del $\mathrm{CRM}$ a condizione che vengano gestiti esclusivamente i clienti più rappresentativi a discapito di una gestione omnicomprensiva di tutte le relazioni.

Sulla base di quanto osservato è possibile affermare che lo sviluppo di questi sistemi potrebbe richiedere sia una profonda conoscenza delle relazioni di business nonché una significativa collaborazione tra le funzioni aziendali coinvolte nello svolgimento delle attività di gestione dei clienti (Lipiäinen, 2015).

Per ciò che concerne l'impatto del CRM sugli utilizzatori del sistema, è importante sottolineare che l'uso del CRM viene fortemente influenzato 
dalle dinamiche organizzative e dalle pressioni che questa subisce dall'ambiente esterno. Inoltre, sarebbero le stesse caratteristiche degli utilizzatori per esempio, il loro background culturale (Härting, Möhring, Schmidt, Reichstein, Keller, 2016) e la propensione all'uso delle tecnologie (PernaBaraldi, 2014) - a creare effetti rispetto i livelli di efficacia dello strumento.

\section{Una nota sulla metodologia}

Da un punto di vista metodologico, il lavoro adotta la strategia del single case study (Yin, 1994) con una prospettiva longitudinale. Questo metodo è stato selezionato in quanto ben supporta le ricerche di tipo esplorativo, ossia quelle focalizzate verso lo studio di processi problematici e complessi da investigare.

La metodologia identificata permette inoltre di investigare specifici eventi (Easton, 1995) riguardanti processi di cambiamento organizzativo all'interno delle imprese (Halinen, Tornroos, 2005).

La raccolta dati è stata condotta nel periodo 2006-2010 - con attività di follow up successive nel periodo 2011-2013 - anche tramite la diretta partecipazione di uno degli autori allo sviluppo dei sistemi CRM presso il gruppo Loccioni, configurandosi quindi come "action research" (Coughlan, Coghlan, 2002). In questo tipo di approccio di ricerca, «l'intervista diventa spesso la fonte primaria dei dati» perché «rappresenta un modo altamente efficiente per raccogliere dati empirici» (Eisenhardt, Graebner, 2007).

Le interviste condotte - complessivamente circa 49 - hanno avuto lo scopo di indagare, nella fase successivamente alla implementazione del CRM, le impressioni degli utenti del sistema riguardo all'utilità del sistema ed eventuali criticità nel suo utilizzo. Interviste semi-strutturate sono state condotte con il top management, i Key Account Managers (KAMs), il responsabile Area Marketing, il responsabile Amministrativo, il responsabile Produzione e tutto il personale dell'area commerciale.

Per studiare l'impatto nell'utilizzo di sistemi CRM nella gestione della relazione è stato selezionato il Gruppo Loccioni per il suo «potenziale rivelatore», in quanto il case study permetterebbe di «acquisire alcune intuizioni che altre organizzazioni non sarebbero in grado di fornire» (Siggelkow, 2007). Inoltre, il caso del gruppo Loccioni risponde appieno alle esigenze di investigare il livello di efficacia del CRM nei casi di imprese che operano in settori complessi come quelli della fornitura di servizi industriali. 


\section{Il caso del gruppo Loccioni}

\subsection{Alcuni cenni introduttivi}

Il gruppo Loccioni, con sede ad Angeli di Rosora (AN), é una impresa che ingegnerizza e sviluppa sistemi e servizi di misura volti al miglioramento dei processi industriali in vari settori quali automotive, elettrodomestico, sanità-medicale. Essi sono sviluppati internamente integrando tecnologie acquistate da partner internazionali; assume fondamentale rilevanza il ruolo svolto dai reparti di ricerca e sviluppo i quali, quindi, sviluppano competenze e know-how per effettuare la suddetta integrazione. L'azienda è stata fondata nel 1968 e nel 2016 ha raggiunto un fatturato di circa 80 milioni di euro impiegando 300 persone.

Le attività produttive e di ricerca sono condotte in Italia e recentemente il gruppo ha incrementato la sua presenza internazionale mediante l'apertura di tre filiali commerciali negli Stati Uniti d'America, in Germania ed in Cina. Le attività di vendite sono condotte principalmente da account managers e da KAMs (Key Account Manager), affiancati dal personale dell'area marketing; la gestione della intera forza vendita è affidata a due figure che svolgono il ruolo di responsabili e determinano insieme al top management gli obiettivi commerciali. L'area marketing, invece, sviluppa attività di tipo strategico volte soprattutto all'identificazione di nuovi clienti ed al presidio dei mercati serviti. In particolare, esiste una forte propensione verso il miglioramento dell'approccio al mercato, potenziando proprio le attività di marketing e quelle commerciali. Intensi sforzi in termini di investimenti sono stati condotti al fine di posizionare l'area marketing a contatto con il top management e con l'area commerciale, in tal senso, risulta molto importante il processo di re-definizione del ruolo del marketing che oggi sembra assumere un ruolo primario nello svolgimento delle seguenti attività operative:

- analisi del mercato attuale;

- ricerche di mercato per valutazione del potenziale;

- analisi dei concorrenti;

- analisi delle innovazioni di prodotti/sistemi e collocazione sul mercato;

- analisi dei costi legati alle politiche di comunicazione (fiere, brochure, manifestazioni, ecc.).

Negli ultimi anni l'impresa ha di fatto modificato il suo modello di business focalizzando l'attenzione anche rispetto l'offerta di servizi ad alto contenuto tecnologico. I clienti possono acquistare non solo le soluzioni tecniche - ad 
esempio macchine e strumentazioni per effettuare attività di misura e collaudo - ma anche servizi accessori di "consulenza" quali auditing dei processi produttivi, oppure reportistiche ad hoc relative alle misurazioni di alcuni parametri critici $^{3}$. Tali attività sono rivolte maggiormente a clienti consolidati ma, secondo il Direttore Generale dell'impresa, l'offerta dei servizi a clienti nuovi potrebbe costituire un promettente mercato da continuare a sviluppare nei prossimi anni. Infatti, tali servizi potrebbero risultare validi per clienti che effettuano notevoli investimenti in attività di ricerca e sviluppo.

Generalmente i clienti serviti sono multinazionali "leader" nei loro rispettivi mercati (si pensi alla Whirlpool e alla Samsung nell'industria dell'elettrodomestico, oppure ai gruppi Continental e Bosch nel settore automotive) che richiedono notevoli sforzi da parte dell'area vendita e marketing del gruppo Loccioni. Infatti l'azienda vende "soluzioni" customizzate dietro richiesta del cliente e non prodotti "standard"; inoltre, la crescente richiesta di servizi accessori ha incrementato il livello di complessità, spingendo il top management ad intraprendere azioni e strategie di marketing mirate e specifiche relativamente ad ogni singola relazione di business.

Per concludere, si evidenzia come nel corso degli anni il gruppo Loccioni abbia venduto le soluzioni in tutti i continenti; la Germania rappresenta, per volumi di fatturato, il paese europeo principale di riferimento. Da sottolineare la grande attenzione che il gruppo ha sempre avuto in America riguardo due mercati: il Messico e gli USA. Le commesse ottenute in tali paesi sono state storicamente legate ai business dell'elettrodomestico e dell'auto.

\subsection{Il processo di implementazione ed utilizzo del CRM}

L'esigenza di sviluppare un sistema CRM matura nel 2006 per iniziativa del Presidente ed a seguito della sua partecipazione ad alcune sessioni formative di marketing e management organizzate da una fondazione bancaria.

Egli considerò interessante il concetto di marketing relazionale ed intraprese - in collaborazione con alcuni consulenti esterni e con la diretta partecipazione del responsabile marketing - un progetto strategico il cui obiettivo finale consisteva nella implementazione di un software CRM. La presenza di competenze tecniche in materia di programmazione e sviluppo di sistemi IT (caratterizzanti la business unit denominata "IT e Telecomunicazione")

\footnotetext{
${ }^{3}$ L'avvio del laboratorio "Kite" rappresenta una delle ultime iniziative condotte dal gruppo Loccioni per potenziare la fornitura di servizi di R\&D.
} 
consentì di sviluppare internamente il sistema. Le principali attività realizzate al fine di svolgere il processo implementativo furono le seguenti:

- indagine interna volta alla comprensione dei metodi di gestione del cliente adottati dal personale dell'area vendita. I risultati evidenziavano chiaramente la carenza di condivisione tra reparti delle informazioni strategiche di mercato; inoltre, ogni intervistato sottolineava la mancanza di strumenti di IT a supporto delle attività commerciali. Risultò necessario riorganizzare la gran parte dei flussi informativi interni con attenzione specifica verso le performance economiche dei clienti (frequenza, quantità, ammontare medio degli acquisti di soluzioni e servizi);

- predisposizione di un piano formativo su temi di CRM e marketing relazionale; circa il 70\% del personale fu coinvolto, nel periodo $2006-$ 2008, compresa la dirigenza aziendale;

- implementazione tecnica del sistema CRM, principalmente eseguita da esperti informatici con la guida ed il supporto dell'area marketing e dei consulenti esterni. Uno sforzo abbastanza notevole venne fatto per permettere al nascente software di poter interagire con gli altri data base e data repository presenti, specialmente quelli utilizzati dal sistema ERP aziendale. Verso le fasi finali fu deciso di dotare il CRM di un cruscotto di controllo di gestione della relazione, ossia furono implementati alcuni indicatori con l'obiettivo di evidenziare le performance economiche e di risultato raggiunte da ogni singolo venditore.

Il sistema fu presentato nel maggio 2008 e dopo 18 mesi venne effettuata una profonda analisi - basata sui risultati di una ricerca condotta con gli utilizzatori del sistema - al fine di verificarne la sua efficacia; emersero diverse problematiche sia dal punto di vista tecnico che socio-organizzativo. Tecnicamente il sistema presentava una sensibile criticità, in quanto non era sufficientemente rapido nel fornire output agli utilizzatori; si evidenziavano con una certa periodicità episodi di blackout del sistema che rendevano impossibile il suo utilizzo. Inoltre, la presenza nel CRM, di informazioni obsolete e spesso ridondanti - ciò dovuto soprattutto al basso livello di commitment degli utenti - contribuì fortemente a limitare la generale e diffusa attitudine all'uso regolare del sistema.

Anche i fattori di natura socio-organizzativa, ossia quelli che riguardano il grado di propensione degli utenti all'uso del software e la loro percezione dei relativi vantaggi, risultarono abbastanza incidenti - purtroppo in maniera negativa - sull'efficacia generale del CRM nel supportare la gestione della relazione con il cliente; basti pensare che si rese necessario approfondire tale 
problematica direttamente con i singoli utenti i quali, in maniera sistematica, manifestarono diverse criticità. Il CRM, infatti, risultava essere uno strumento appropriato maggiormente per la gestione di relazioni con clienti nuovi affidati a junior account managers; al contrario, risultò superfluo relativamente al controllo ed al coordinamento di relazioni storiche affidate a senior key account managers.

Infine, un altro elemento discordante risultò essere la diffusa percezione che il sistema non fosse in grado di tracciare appieno le dinamiche interorganizzative (ossia gli eventi e gli episodi rilevanti che caratterizzano le relazioni con i clienti) costituendo, quindi, un problema dal punto di vista della sua utilità per una parte degli utilizzatori. Allo stesso tempo alcuni utilizzatori sottolineavano che il CRM fosse maggiormente deputato a svolgere funzioni di controllo delle performance, piuttosto che fornire conoscenza per migliorare la gestione della relazione di business.

\section{Analisi del caso}

L'analisi del caso empirico si pone come principale obiettivo quello di rispondere alle domande di ricerca formulate nella sezione introduttiva: (1) quali sono i fattori che influenzano l'efficacia del CRM in mercati caratterizzati dallo scambio di servizi tecnologici? (2) Come varia la percezione degli utilizzatori del CRM rispetto la sua efficacia?

In riferimento al primo quesito, è abbastanza evidente come siano molteplici ed eterogenei i fattori che influenzano il livello di efficacia del CRM. In primo luogo, lo sviluppo del progetto CRM nel gruppo Loccioni è stato caratterizzato dal tentativo di implementare uno strumento volto al miglioramento di alcuni processi di gestione del cliente. Ma uno dei principali motivi che hanno giustificato l'avvio del processo di implementazione riconduce al tentativo di emulare (Hillebrand et al., 2011) simili practice aziendali intraprese da altre organizzazioni; infatti, solo quando il Presidente ha partecipato a momenti formativi riceve lo stimolo a raccogliere maggiori informazioni inerenti i benefici del processo CRM.

Il processo di implementazione costituisce un altro importante elemento che interviene ed influenza notevolmente l'efficacia del sistema. L'intento dell'azienda è quello di sviluppare internamente una piattaforma CRM in virtù delle competenze maturate dalla business unit "IT e Telecomunicazioni". Il periodo di sviluppo della piattaforma procede, però, in maniera discontinua a causa di alcune problematiche inaspettate che derivano da una 
sostanziale mancanza di coinvolgimento degli utenti chiave, ossia i key account managers. Se ne deduce che l'implementazione creerebbe effetti tangibili sull'efficacia stessa e nel caso preso in esame, rivelatisi non sempre positivi.

Inoltre, l'introduzione del CRM nell'organizzazione ha creato effetti sulle altre tecnologie già presenti. Il CRM è stato sviluppato sulla base di un pre-esistente data base marketing, ma ha necessitato diversi adattamenti tecnici, che hanno condotto alla creazione di interfacce tecniche (Baraldi, 2003). Tali interfacce sono la testimonianza della complessità del processo tecnico di sviluppo, di implementazione ed uso del CRM. Infatti, le interfacce tecniche (per esempio tra il CRM e gli altri data repository) modellano sia i processi di inserimento dei dati, ma soprattutto impattano sul tipo di conoscenza che l'utente ottiene dall'interazione con il CRM stesso.

Un altro elemento considerevole si riconduce all'idea che il CRM possa agevolare, comunque, l'operato degli user intenti ad affrontare la "quotidiana" gestione del mercato. In realtà, il caso evidenzia come il livello di soddisfazione degli utenti sia dipendente dal tipo di relazione con il cliente (Perna-Baraldi, 2014) e dalla predisposizione del key account manager ad utilizzare le tecnologie per controllare e gestire aspetti strategici della relazione di business.

I risultati indicano in maniera abbastanza esplicita che l'efficacia di un sistema CRM dipende sia da aspetti puramente tecnici (funzionalità e facilità di utilizzo del software, nonché assenza di errori nella programmazione), ma soprattutto da quelli socio-organizzativi. A questo proposito, rispondendo al secondo quesito della nostra ricerca, l'analisi del caso offre interessanti chiavi di lettura circa i motivi principali che influenzano le percezioni degli utenti del CRM per quanto concerne la sua efficacia.

La complessità dell'offerta e la presenza di numerosi attori coinvolti nei processi di business - caratteristiche principali dei mercati industriali - dovrebbero favorire positivamente la percezione che il CRM sia uno strumento utile ed a supporto delle attività di gestione dei processi di marketing. Ciò in quanto l'uso delle tecnologie, in generale, favorirebbe l'operato del management aziendale circa lo svolgimento di processi di decision making; si pensi, per esempio, alla tracciabilità delle diverse fasi della relazione con il cliente (Ford, 1980). Ma evidenze empiriche dimostrano anche che gli utenti non sono sempre propensi ad utilizzare il software, in quanto potrebbero prediligere l'uso di pratiche più flessibili e meno strutturate. Si ravvisa invece, per tipologie di offerta meno complesse, ossia quelle relative alle vendite di "prodotti" o "soluzioni" standard e per l'assistenza tradizionalmente intesa 
come "attività post-vendita", una utilità percepita maggiore e quindi maggiori benefici, che si traducono in un'adozione più veloce ed immediata delle pratiche legate all'utilizzo dei software CRM. A conclusione di quanto affermato, la percezione dell'efficacia del CRM potrebbe dipendere sia da variabili non direttamente controllabili (per esempio, le forze e le strutture di mercato) nonché dalla predisposizione degli utenti verso l'uso della tecnologia come strumento di lavoro e dalla presenza di un ambiento organizzativo favorevole ad accettare le tecnologie stesse.

\section{Conclusioni}

Nel presente lavoro il tema del CRM è stato affrontato con il principale obiettivo di comprendere quali siano i principali fattori che ne influenzano l'efficacia - intesa come l'insieme dei benefici che gli utilizzatori potrebbero ottenere dal suo impiego. Inoltre, il lavoro ha descritto ed analizzato il rapporto tra l'efficacia del CRM e le aspettative dei suoi utilizzatori e, a livello generale, di tutta l'organizzazione.

L'esame del caso empirico presentato, basato sull'esperienza del gruppo Loccioni, consente di osservare la complessità e la non previsione degli effetti in termini di efficacia che l'uso di tali sistemi genera. In particolar modo, si rileva come sia abbastanza complicato cercare di misurare l'efficacia del sistema in maniera univoca. L'interazione tra user e sistema CRM è fortemente variabile e di conseguenza anche l'efficacia varia sensibilmente; laddove la gestione delle relazioni con clienti nuovi è affidata a young key account managers sembrerebbe che il CRM possa generare dei benefici. Al contrario, nei casi in cui le relazioni storiche sono gestite da senior key account managers si è notato un sensibile inutilizzo del sistema e quindi un'efficacia limitata.

In maniera più generale, l'analisi del caso ha consentito di rilevare che $\mathrm{i}$ singoli users abbiano differenti aspettative e percezioni circa l'utilità del CRM, non prevedibili. Solo alcuni gruppi di users, mostrano predisposizione verso l'uso di un sistema che digitalizza le relazioni di business; le ragioni sono intuibili e sono connesse alla stessa natura della customer relationship, che sottende la presenza di una sostanziale complessità dovuta ai complicati meccanismi interattivi. Inoltre, l'efficacia del sistema sembra si leghi anche al corretto processo di implementazione ed al pieno coinvolgimento delle varie funzioni (marketing e vendite, principalmente) nelle attività di inserimento dati e nell'uso delle informazioni ricevute come output. 
Il paper affronta in maniera originale il tema del CRM e della sua efficacia e fornisce alcuni contributi teorici ed empirici. Dal punto di vista empirico, il CRM è investigato in un contesto quale quello dell'offerta di soluzioni e servizi complessi in mercati industriali; sono abbastanza rari studi longitudinali sviluppati con la strategia action research (Coughlan-Coghlan, 2002), soprattutto considerando la prospettiva della media impresa italiana. Altro contributo empirico è evidenziabile in relazione allo studio del processo di utilizzo del CRM da un punto di vista socio-tecnico. Per quanto concerne la prospettiva teorica, il presente studio sviluppa ulteriormente i risultati ottenuti in precedenti studi (Baraldi, La Rocca, Perna, 2013; Perna-Baraldi, 2014), analizzando il tema dell'efficacia del CRM in termini di dimensione fortemente influenzata sia dalle caratteristiche delle relazioni di business che dalle dinamiche intra-organizzative.

Si vuole concludere offrendo alcuni spunti di riflessione circa le implicazioni manageriali e possibili ambiti di ricerca sul tema efficacia-CRM. Le imprese che intendono dotarsi di sistemi CRM dovrebbero essere consapevoli che l'efficacia derivante dall'uso del sistema andrebbe declinata e comunicata agli utilizzatori; inoltre, il coinvolgimento del personale che opera sul mercato appare fondamentale sin dalle iniziali fasi implementative. Altro elemento ritenuto importante si riscontra nel rendere il sistema flessibile ed adattabile in modo che esso possa effettivamente digitalizzare le complesse "parti" della relazione di business. Di conseguenza, si renderebbe necessario individuare un CRM manager che possa assicurare il corretto funzionamento tecnico ed organizzativo del sistema e che possa sviluppare attività formative dirette alla totalità degli utilizzatori.

Circa le prospettive di ricerche future sul rapporto tra CRM, efficacia e percezioni degli utilizzatori, si vuole suggerire l'importanza di effettuare analoghi studi in organizzazioni di settori (per esempio, contesti di mercato business-to-consumer) e dimensioni (imprese start-up, imprese micro e multinazionali), differenti rispetto al caso preso in esame. Da ultimo, gli autori suggeriscono di sviluppare studi anche di tipo quantitativo, identificando i possibili KPI dell'efficacia del sistema CRM. 


\section{Riferimenti bibliografici}

Alshawi, S., Miss, F., e Irani, Z., Organisational, Technical and Data Quality Factors in CRM Adoption - SME's perspective, in «Industrial Marketing Management Journal», 2011, pp. 376-383.

Avlonitis, G.J., e Panagapoulos, N.G., Antecedents and consequences of CRM technology acceptance in sales force, in «Industrial Marketing Management», 2005, pp. 355-368.

Baraldi, E., La Rocca, A., e Perna, A., Intra- and Inter-organizational effects of a CRM system implementation, «Mercati e Competitività», 2013, pp. 13-34.

Baraldi, E., When Information technologies faces resource interaction. Doctoral Thesis. Uppsala, Uppsala University, Business Studies Department, 2003.

Berry, L.L., Relationship marketing, in Perspective on Services, a cura di L.L. Berry, G.L. Shostack, e G.D. Upah, Chicago, Marketing American Marketing Association, 1983.

Boulding, W., Staelin, R., Ehret, M., e Johnston, W.J., A CRM roadmap: What we know, potential pitfalls, and where to go, in «Journal of Marketing», 2005, pp. $155-166$

Bucic, T., Ngo, L.V., e Sinha, A., Improving the effectiveness of market-oriented organisation: Empirical evidence from an emerging economy, in «Australian Journal of Management», 2017, pp. 308-327.

Bull, C., Strategic issues in customer relationship management (CRM) implementation, in «Business Process Management Journal», 2003, pp. 592-602.

Cambra-Fierro, J.J., Centeno, E., Olavarria, A., e Vasquez-Carrasco, R., Success factors in a CRM strategy: technology is not all, in «Journal of Strategic Marketing», 2017, pp. 316-333.

Chang, S.I., Hung, S.Y., Yen, D.C., e Lee, P.J., Critical factors of ERP Adoption for Small-and-Medium-Sized Enterprises: An Empirical Study, in «Journal of Global Information Management», 2010, pp. 82-106.

Coughlan, P., e Coghlan, D. Action research for operation management, in «Internationa Journal of Operations \& Production Management», 2002, pp. 230-240.

Cunningham, M., e Turnbull, P.W., Inter-Organizational Personal Contact Patterns, in International Marketing and Purchasing of Industrial Goods: An Interaction Approach, a cura di H. Håkansson, Chichester, Wiley, 1982.

Easton, G., Case research as a methodology for industrial networks; a realist apologia, Lancaster, 1995.

Eisenhardt, K.M., e Graebner, M.E., Theory building from cases: opportunities and challenges, in «Academy of management journal», 2007, pp. 25-32.

Elmuti, D., Jia, H., e Gray, D., Customer relationship management strategic application and organizational effectiveness: An empirical investigation, in «Journal of Strategic Marketing», 2009, pp. 75-96.

Fiocca, R., Account Portfolio Analysis for Strategy Development, in «Industrial Marketing Management», 1982, pp. 53-62.

Ford, D., The Development of Buyer-Seller Relationships in Industrial Markets, in «European Journal of Marketing», 1980, pp. 339-353. 
Ford, D., e Håkansson, H., How should companies interact in business networks?, in «Journal of Business Research», 2002, pp. 133-139.

Ford, D., e Rosson, P.J., The Relationship Betweeen Export Manufacturers and their Overseas Distributors, in Export Management: An International Context, a cura di M. Czinkota, New York, Praeger Publishers, 1982.

Ford, D., Gadde, L.-E., Håkansson, H., e Snehota, I., The Business Marketing Course. Managing in Complex Networks, Chichester, Wiley, 2006.

Ford, D., Gadde, L.-E., Håkansson, H., e Snehota, I., Managing Business Relationship, Third Edition, Chichester, John Wiley \& Sons, 2011.

Gadde, L.-E., Hjelmgren, D., e Skarp, F., Interactive resource development in new business relationship, in «Journal of Business Research», 2012, pp. 210-217.

Garrido-Moreno, A., Lockett, N.J., e Morales, V., Paving the way for CRM success: the mediating role of knowledge management and organizational commitment, in «Information \& Management», 2014, pp. 1031-1042.

Gronroos, C., From Marketing Mix to Relationship Marketing: Towards a Paradigm Shift in Marketing. Management Decision, 1994, pp. 4-20.

Gummesson, E., Lehtinen, U., e Gronroos, C., Nordic perspectives on relationship marketing, in «European Journal of Marketing», 1997, pp. 10-16.

Härting, R.-C., Möhring, M., Schmidt, R., Reichstein, C., e Keller, B., What drives user to use CRM in a Public Cloud environment? - Insights from European Experts, Hawaii International Conference on System Sciences, 2016, pp. 39994008.

Håkansson, H., Ford, D., Gadde, L.-E., Snehota, I., e Waluszewski, A., Business in Networks, Chichester, John Wiley \& Sons, 2009.

Håkansson, H., e Snehota, I., Developing Relationships in Business Network, London, Routledge, 1995.

Halinen, A., e Tornroos, J., Using case methods in the study of contemporary business networks, in «Journal of business research», 2005, pp. 1285-1297.

Hillebrand, B., Nijholt, J.J., e Nijssen, E.J., Exploring CRM effectiveness: an institutional theory perspective, in «Journal of the Academy of Marketing Science», 2011, pp. 592-608.

Kalaignanam, K., e Varadarajan, R., Offshore outsourcing of customer relationship management: conceptual model and propositions, in «Journal of the Academy of Marketing Science», 2012, pp. 347-363.

La Rocca, A., Ford, D., e Snehota, I., Initial relationship development in new business ventures, in «Industrial Marketing Management», 2013, pp. 1025-1032.

Lipiäinen, H., CRM in the digital age: implementation of CRM in three contemporary B2B firms, in «Journal of Systems and Information Technology», 71, 2015, 1, pp. 2-19.

Payne, A., e Frow, P., A Strategic Framework for Customer Relationship Management, in «Journal of Marketing», 2005, pp. 167-176.

Perna, A., L'implementazione del Customer Relationship Management in contesti Business-to-Business, Bologna, Esculapio, 2013.

Perna, A., e Baraldi, E., CRM Systems in Industrial Companies: Intra- and InterOrganizational Effects. Palgrave Macmillan, 2014. 
Runfola, A., Perna, A., Baraldi, E., e Gregori, G., The use of qualitative case studies in top business and management journals: A quantitative analysis of recent patterns, in «European Management Journal», 2016, pp. 1-12.

Siggelkow, N., Persuasion with case studies, in «Academy of Management Journal», 2007, pp. 20-24

Snehota, I., Håkansson, H., Gadde, L.-E., e Ford, D., Managing Business Relationships, Chichester, Wiley, 2003.

Stone, M., Woodcock, N., e Wilson, M., Managing the change from marketing planning to customer relationship management, in «Long Range Planning», 1996, pp. 675-683.

Turnbull, P.W., e Zolkiewski, J.M., Profitability in Customer Portfolio Planning, in Understanding Business Markets, a cura di D. Ford, London, Dryden Press, 1997.

Tzafilkou, K., Protogeros, N., e Yakinthos, C., End-user development of CRM system: towards a behavioural end-user profiling based on gender and expertise, in «International Journal of Electronic Businesses», 2015.

Wongsansukcharoen, J., Trimetsoontorn, J., e Fongsuwan, W., Social CRM, RMO and business strategies affecting banking performance effectiveness in B2B context, in «Journal of Business \& Industrial Marketing», 30, 2015, 6, pp. 742-760.

Yin, R.K., Case study research: Design \& Methods, Newbury Park, CA, Sage, 1994.

Zablah, A.R., Bellenger, D.N., e Johnston, W.J., An evaluation of divergent perspectives on customer relationship management: Towards a common understanding of an emerging phenomenon, in «Industrial Marketing Management», 2004, pp. 475-489. 